\title{
IN SITU DIGITAL IMAGE SPECKLE CORRELATION (DISC) OBSERVATION OF PLASTIC STRAIN INCREMENT IN LOW-CARBON STEEL
}

\author{
Received - Primljeno: 2020 -06-25 \\ Accepted - Prihvaćeno: 2020-10-15 \\ Original Scientific Paper - Izvorni znanstveni rad
}

The present work is aimed at studying the macroscopic localization of plastic strain in low-carbon steel AISI A283 Grade C. The evolution of macroscopically localized plastic strain at various stages of strain hardening is visualized via a Digital image speckle correlation (DISC) method. The processing of stress-strain curves of the steel samples enabled one to distinguish the following stages of strain hardening: the yield point, the parabolic hardening, and the pre-failure. The inspection of local strain distributions reveals that the parabolic work hardening is presented by a system of stationary plastic strain centers placed at equal distances. To perform a comprehensive analysis of local elongations from the parabolic work hardening toward the pre-failure stage, the total elongation components were measured at the strain centers. The average total elongation is found to be almost constant at the parabolic work hardening, but abruptly increases when approaching the pre-failure stage. The plastic strain instability is highlighted, as well, by the rising total elongation (amplitude) at the strain sites.

Key words: low-carbon steel, localization of the plastic deformation, DISC, tensile test, strain

\section{INTRODUCTION}

The origin of macrolocalization and strain hardening that act at specific strain stages remains still poorly understood from the physical viewpoint of plastic flow when passing from one stage to another. In spite of the fact that strain hardening mechanisms themselves at certain strain stages have been sufficiently described at the macroscopic level [1], the reasons of the transition between various strain stages remain an open question. Furthermore, the correlation between microscopic mechanisms and macroscopically localized plastic strain is still under discussion [2-4]. Thus there are some gaps in both microscopic and macroscopic descriptions of the phenomenon of plasticity. On the one hand this impedes the complete understanding of materials hardening, but on the other it inhibits the development of technological processing methods involving severe plastic strain. It is known that plastic strain tends to localize at all stages of flow [5-7] and takes its origin from the nucleation and propagation of the Lüders (LB) or Portevin-Le Chatelier bands (PLC) [7-9]. A comparative analysis was made of the experimental data obtained for a wide range of materials above, which allows for identification of plastic flow features common to all materials. The localization behavior of plastic deformation is its most salient feature. By constant-rate

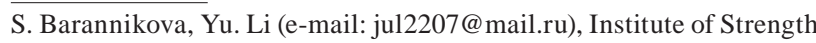
Physics and Materials Science of Siberian Branch Russian Academy of Sciences (ISPMS SB RAS), Tomsk, Russia; National Research Tomsk State University, Tomsk, Russia; Tomsk State Architecture and Building University, Tomsk, Russia
}

tensile loading, space-time periodic structures, so-called deformation patterns, emerge in the deforming sample from the yield limit to its failure. Quantitatively the deformation patterns are characterized by spatial, $\alpha \approx 10^{-2}$ $\mathrm{m}$, and temporal, $T \approx 10^{2} \mathrm{~s}$, scales and are propagated with rate $10^{-5} \leq \mathrm{V} \leq 10^{-4} \mathrm{~m} / \mathrm{s}$. The following features are common to all the localization patterns observed thus far:

- localization structures will occur spontaneously in the sample by constant-rate loading in the absence of any specific action from the outside;

- in the course of plastic deformation a changeover in the types of localized plasticity patterns is observed;

- the emergence of such patterns is unrelated to deformation micromechanisms;

- due to work hardening, the deforming medium's defect structure undergoes irreversible changes, which are reflected in the emergent patterns and are suggestive of its non-linearity.

The well-known LB front can be regarded as a boundary between the elastically and plastically deforming material volumes. As the Lüders front propagates along the tensile sample, it leaves behind an ever increasing volume of deformed material [1]. Due to the structural changes, the deforming material volume acquires a new state, which is characterized by increasing density of defects; its deformation occurs via dislocation glide mechanism. With growing total deformation, the plastic flow will exhibit an intermittent behavior on the macro-scale level. But the LB macroscopic strain bands may be due to technological defects during the 
manufacture of low-carbon steel sheets, because such deteriorate the quality of the products and induce premature corrosion and failure. Hence localized plastic strain has to be thoroughly studied at the different stages of plastic flow [10-14]. In this respect, the present work is aimed at investigating the macroscopic strain localization in low-carbon steel ASTM A 283 Grade C.

\section{EXPERIMENTAL}

\section{Materials and fabrications}

As-prepared double shoulder-like samples with working areas of $50 \times 10 \times 2 \mathrm{~mm}$ were stretched at $\mathrm{T}=300 \mathrm{~K}$ at a rate of $6,67 \times 10^{-5} \mathrm{~s}^{-1}$ using a LFM-125 testing machine. The microstructure of low-carbon steel ASTM A 283 Grade C is a ferrite matrix with a small amount of perlite grain inclusions with average grain sizes of $10 \pm$ $4,5 \mu \mathrm{m}$. The evolution of macroscopically localized plastic strain bands at the different stages of strain hardening was visualized via a digital image speckle (DISC) correlation method [15]. For this, the stretched sample was illuminated with a coherent beam of a semiconductor laser with a wavelength of $635 \mathrm{~nm}$ and a power of $15 \mathrm{~W}$. The images of the deformed sample under illumination with superposed speckle structures were recorded at a frequency of $10 \mathrm{~Hz}$ with a pixellink PL-B781 digital video camera, digitized and saved as files. The digital image speckle correlation consists in the high-accuracy determination of shear fields via monitoring changes across the specimen surface. The digital images obtained during uniaxial stretching are then subjected to processing.

\section{RESULT AND DISCUSSION}

The analysis of the stress-strain curves of ASTM A 283 Grade C steels allowed one to distinguish the ensuing stages of work hardening. First, the transition area from the elasticity toward the plastic flow at the total strain $\varepsilon_{\text {tot }}=0,014-0,03$ is followed by the emergence of the yield point. After the plastic flow, there is the parabolic strain (Taylor) hardening at $\varepsilon_{\text {tot }}=0,1-0,23$. Finally, at $\varepsilon_{\text {tot }}=0,23-0,28$ it observes the pre-failure stage with a sample failure and the neck formation.

In obedience to the experimental data, plastic strain localization (PSL) in low-carbon steel originates from the Lüders band, which is manifested by a slight increase in stress with a subsequent rapid decrease in the stress-strain curve. At that moment, the first PSL single front arises at a mobile clutch at the total strain $\varepsilon_{\text {tot }}=$ 0,008 and the angle of $70 \mathrm{deg}$. Despite the front is $\sim 1$ $\mathrm{mm}$ large, it exhibits neither further increase nor propagation, almost vanishing at the strain $\varepsilon_{\text {tot }}=0,009$ because of its departure from the visible part of the sample. When achieving the strain corresponding to the upper yield strength $\sigma_{T}^{B}=265 \mathrm{MPa}$ and the total strain $\varepsilon_{\text {tot }}=$ 0,014, there is the emergence of the second PSL Lüders band at the upper stationary clutch of the testing ma-

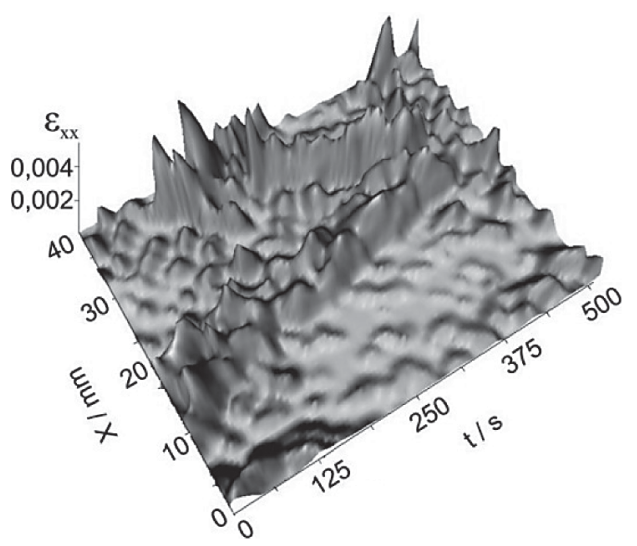

(a)

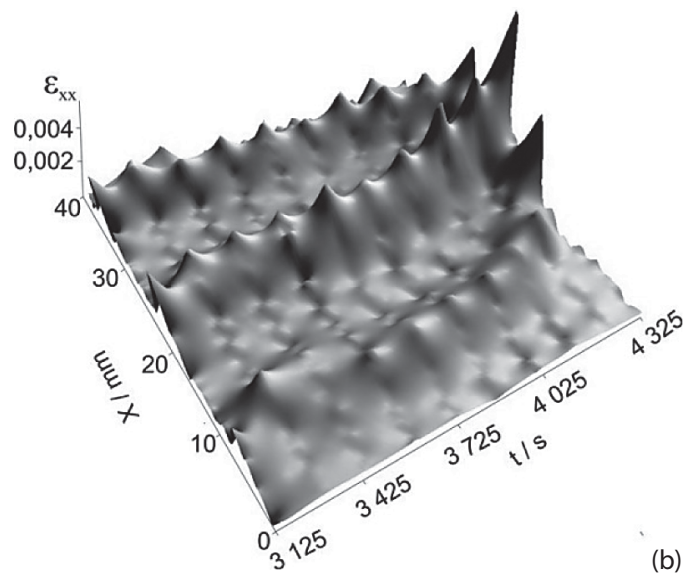

Figure 1 Plastic strain localization pattern of ASTM A 283 Grade C sample: (a) at the yield flow (1 and 2 - LSB) and at the parabolic work hardening stage (b) where: $x$ - lengths of specimen, $t$ - time of loading, $\varepsilon_{x x}$ - local elongations

chine at an angle of $60 \mathrm{deg}$, which then spreads across the sample at a stress $\sigma_{T}^{H}=260 \mathrm{MPa}$. Besides the presence of the yield drop throughout the yield point, it observes the slight stress variations associated with the formation and instaneous disappearance of additional bands in undeformed parts of the samples, which, however, can be descried at the mobile clutch of the machine. At the same time, at the total strain $\varepsilon_{\text {tot }}=0,019$ there is the appearance of the second Lüders band at the stationary clutch of the machine, and both bands move toward each other.

Figure 1a shows the evolution of plastic strain centers within the yield flow as two LSB (Lüders strain bands) moving toward each other with velocities $V_{1} \approx$ $1,1 \cdot 10^{-4} \mathrm{~m} / \mathrm{s}$ and $V_{2} \approx 0,6 \cdot 10^{-4} \mathrm{~m} / \mathrm{s}$. The meeting of the described localization centers results in their mutual annihilation at the end of the yield flow at the strain $\varepsilon_{\text {tot }}$ $=0,031$.

According to the analysis of local strain distributions, low-carbon steels ASTM A 283 Grade C undergoing the parabolic hardening stage at the total strain $\varepsilon_{\text {tot }}$ $=0,1-0,23$ possess three stationary plastic strain localization sites arranged at equal distances with a spatial period $\lambda=7 \pm 1 \mathrm{~mm}$. At the pre-failure stage $\left(\varepsilon_{\mathrm{tot}}=0,23\right.$ $-0,28$ ) these sites gradually move to the high-amplitude local elongation maximum (Figure 1b) The inspection of the kinetics of localized plasticity sites with increa- 


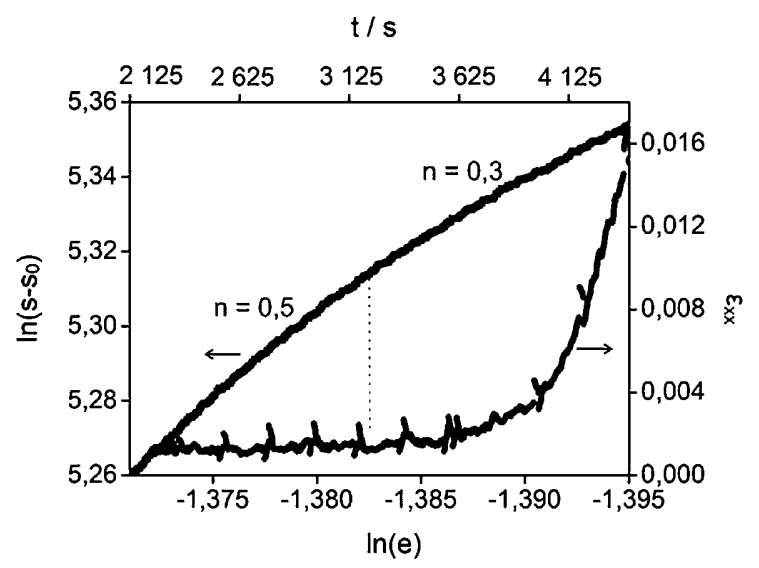

Figure 2 Plastic strain increment $\varepsilon_{x x}$ in ASTM A 283 Grade C during the parabolic hardening $(n=0,5)$ and pre-failure stage $(n=0,3)$

sing total strain reveals their merge at the nucleation center of a viscous crack, arriving almost simultaneously at this sample section. Such a strain pattern is typical of the stage preceding the beginning of the fall of conditional stresses in the $\sigma(\varepsilon)$ diagram, i.e. in range of $\sigma_{*}<\sigma<\sigma_{B}$, where $\sigma_{*}$ is the stress associated with the end of the parabolic work hardening and $\sigma_{B}$ is the ultimate stress.

For a comprehensive analysis of local elongations $\varepsilon_{\mathrm{xx}}$ during the transition from the parabolic work hardening to the pre-failure stage, the total values of $\varepsilon_{x x}$ components were measured for the different points along the loading axis of the sample at $\varepsilon_{\text {tot }}=0,15-0,28$. It is found that the average total elongation $\varepsilon_{\mathrm{xx}}$ is almost constant at the parabolic hardening, but abruptly increases when reaching the strain center at $\varepsilon_{\text {tot }} \sim 0,19-$ 0,25 , which corresponds to the transition from the parabolic strain hardening to the pre-failure stage (Figure 2).

The extreme increase in the total elongation (Figure 2) is testimony to the fact that from all plastic strain foci only one retains its activity with time and its position upon nucleation matches the future nucleation center of the macroscopic neck and the viscous failure, respectively. Arising during the parabolic work hardening (Figure 1b), this foci remains quasi-stationary up to the failure, but strain in it gradually rises with attenuating plastic strain activity in other centers.

\section{CONCLUSION}

- Applying digital image speckle correlation and static digital image speckle methods was shown to allow a thorough real-time study of macroscopic strain localization upon uniaxial stretching and the distinction of various plastic flow stages in low-carbon steels ASTM A 283 Grade C.

- The local strain distributions $\varepsilon_{x x}$ in low-carbon steel ASTM A 283 Grade C at the yield flow were found to be presented by the two localized strain zones (Lüders bands). The parabolic work hardening is characterized by the formation of a stationary system of three strain localization zones arranged at equal distances with a spatial period of $7 \pm 1 \mathrm{~mm}$.

- The failure of low-carbon steel is a result of preliminary plastic flow processes. This point of view coincides with statements [16], according to which plasticity and failure are causally correlated, whereas failure is the final stage of plastic strain. The merge zone of plasticity centers traveling at the pre-demolition stage has a complex structure. The formation of the neck and the transition from the plastic flow to the viscous failure during the pre-failure take place when the distance between the plasticity centers gradually and naturally decreases, leading to their merge into the high-amplitude one. At this stage, there are no more signs of plastic flow processes in the whole volume of the sample, except for the part being directly adjacent to the neck.

- The plastic deformation increment is highlighted, as well, which is characterized by the exponential increase in the total (amplitude) elongation $\varepsilon_{x x}$ during the transition from the parabolic work hardening to the prefailure in the neck formation region.

\section{Acknowledgments}

This work was supported in the framework of the Program of fundamental scientific research of Russian State Academies of Sciences for 2013-2020 (direction III.23.1.2) and the Program of Competitiveness by the National Research Tomsk State University.

\section{REFERENCES}

[1] S. D. Antolovich, R. W. Armstrong. Plastic strain localization in metals: origins and consequences. Progress in Materials Science 59 (2014), 1-160.

[2] A. Ziegenbein, P. Hähner, H. Neuhäuser. Correlation of temporal instabilities and spatial localization duringPortevin-Le Châtelier deformation of $\mathrm{Cu}-10$ at.\% $\mathrm{Al}$ and Cu-15 at.\% Al. Computational Materials Science 19 (2000) 1, 27-34.

[3] J. Min, L. G. Hector, J. E. Carsley, T. B. Stoughton, B. E. Carlson, J. Lin. Spatio-temporal characteristicsof plastic instability in AA5182-O during biaxial deformation. Materials and Design 83 (2015), 786-794.

[4] Y.L. Cai, S.L. Yang, Y.H. Wang, S.H. Fu, Q.C. Zhang. Characterization of the deformation behaviors associated with the serrated flow of a $5456 \mathrm{Al}$-based alloy using two orthogonal digital image correlation systems. Materials Science and Engineering: A 664 (2016), 155-164.

[5] S. Graff, S. Forest, J.-L. Strudel, C. Prioul, P. Pilvin, J.-L. Bechade. Strain localization phenomena associated with static and dynamic strain ageing in notched specimens: experiments and finite element simulations. Materials Science and Engineering: A 387 (2004), 181-185.

[6] J. Kang, D.S. Wilkinson, M. Jain, J.D. Embury, A.J. Beaudoin, S. Kim, R. Mishira, A.K. Sachdev. On the sequence of inhomogeneous deformation processes occurring during tensile deformation of strip cast AA5754. Acta Materialia 54 (2006), 209-218.

[7] M. A. Lebyodkin, D. A. Zhemchuzhnikova, T. A. Lebedkina, E.C. Aifantis. Kinematics of formation and cessation of type B deformation bands during the Portevin-Le Chatelier 
effect in an AlMg alloy. Results in Physics 12 (2019), 867-869.

[8] B. Reyne, P. Manach, N. Moës. Macroscopic consequences of Piobert-Lüders and Portevin-Le Chatelier bands during tensile deformation in Al-Mg alloys. Materials Science and Engineering A 746 (2019) 187-196.

[9] H. Ait-Amokhtar, P. Vacher, S. Boudrahem. Kinematics fields and spatial activity of Portevin-Le Chatelier bands using the digital image correlation method. Acta Materialia 54 (2006), 4365-4371.

[10] B. Swaminathan, W. Abuzaid, H. Sehitoglu, J. Lambros. Investigation using digital image correlation of PortevinLe Chatelier Effect in Hastelloy X under thermo-mechanical loading. International Journal of Plasticity 64 (2015), 177-192.

[11] X. G. Wang, L. Wang, M. X. Huang. Kinematic and thermal characteristics of Lüders and Portevin-Le Châtelierbands in a medium Mn transformation-induced plasticity steel. Acta Materialia 124 (2017) 17-29.

[12] S. A. Barannikova, A. V. Ponomareva, L. B. Zuev, Yu. Kh. Vekilov, I. A. Abrikosov. Significant correlation between macroscopic and microscopic parameters for the descrip- tion of localized plastic flow auto-waves in deforming alloys. Solid State Communications. 152 (2012), 784-787.

[13] D. V. Orlova, S. A. Barannikova, L. B. Zuev. On the kinetics of localized plasticity domains emergent at the prefailure stage of deformation process. AIP Conference Proceedings 1783 (2016), 020168(1) - 020168(4) .

[14] L. B. Zuev, S. A. Barannikova, O. A. Maslova. The features of localized plasticity autowaves in solids. Journal of Materials Research 22 (2019), e20180694.

[15] L. B. Zuev, V. V. Gorbatenko, K. V. Pavlichev. Elaboration of speckle photography techniques for plastic flow analyses. Measurement Science and Technology 21 (2010), 054014(1) - 054014(5).

[16] G. V. Kurdyumov, N. V. Klassen-Neklyudova. Progress in the theory of strength and plasticity of solids (A review of A. V. Stepanov's works). Soviet Physics Uspekhi. 16 (1974), 828-833.

Note: The response for English language is YU.V Stankina the translation professional of National Research Tomsk State University «TSU» 